\title{
Effect of N-Terminal Glutamic Acid and Glutamine on Fragmentation of Peptide Ions
}

\author{
Bhaskar Godugu, Pedatsur Neta, Yamil Simón-Manso, and \\ Stephen E. Stein
}

Chemical and Biochemical Reference Data Division, National Institute of Standards and Technology, Gaithersburg, Maryland, USA

\begin{abstract}
A prominent dissociation path for electrospray generated tryptic peptide ions is the dissociation of the peptide bond linking the second and third residues from the amino-terminus. The formation of the resulting $b_{2}$ and $y_{n-2}$ fragments has been rationalized by specific facile mechanisms. An examination of spectral libraries shows that this path predominates in diprotonated peptides composed of 12 or fewer residues, with the notable exception of peptides containing glutamine or glutamic acid at the N-terminus. To elucidate the mechanism by which these amino acids affect peptide fragmentation, we synthesized peptides of varying size and composition and examined their MS/MS spectra as a function of collision voltage in a triple quadrupole mass spectrometer. Loss of water from N-terminal glutamic acid and glutamine is observed at a lower voltage than any other fragmentation, leading to cyclization of the terminal residue. This cyclization results in the conversion of the terminal amine group to an imide, which has a lower proton affinity. As a result, the second proton is not localized at the N-terminus but is readily transferred to other sites, leading to fragmentation near the center of the peptide. Further confirmation was obtained by examining peptides with $\mathrm{N}$-terminal pyroglutamic acid and N-acetyl peptides. Peptides with N-terminal proline maintain the trend of forming $b_{2}$ and $y_{n-2}$ because their ring contains an imine rather than imide and has sufficient proton affinity to retain the proton at the N-terminus. (J Am Soc Mass Spectrom 2010, 21, 1169-1176) (C) 2010 American Society for Mass Spectrometry
\end{abstract}

$\mathrm{P}$ eptide ion fragmentation by tandem mass spectrometry has become a routine means of determining peptide sequence for the purpose of protein identification [1]. A prominent dissociation path for electrospray-generated tryptic peptide ions is the breaking of the peptide bond linking the second and third residues from the amino-terminus [2-6]. The formation of the resulting $b_{2}$ and $y_{n-2}$ fragments from diprotonated peptide ions (with $\mathrm{n}$ amino acid residues) has been rationalized by specific facile mechanisms [2]. It has been pointed out, however, that certain peptides ions do not fragment preferentially by this route but rather fragment at peptide bonds closer to the center of the peptide [2]. To explain the difference between the two groups of peptides it was suggested that the dominant $b_{2}$ ions formed from the first group have a protonated diketopiperazine structure (1) whereas $b_{2}$ ions from the other peptides have a protonated oxazolone structure (2).

Address reprint requests to Dr. S. Stein, NIST Mass Spectral Data Center, Chemical and Biochemical Reference Data Division, Gaithersburg, MD 20899, USA. E-mail: steve.stein@nist.gov<smiles>[R]C1NC(=O)C([R])NC1=O</smiles>

1<smiles>[R]C(N)C1=NC([R2])C(=O)O1</smiles>

2
More recent studies, however, indicated that $b_{2}$ ions from the first group also have the oxazolone structure $[5,6]$ so that the reason for the difference between the two groups remains unclear. Another recent study of several synthetic $(\mathrm{Ala})_{\mathrm{x}}$ His peptide ions indicated that this fragmentation pathway diminished with increasing peptide length [4].

In the present study, we attempt to distinguish between these two groups of peptides by searching for correlation between the mode of fragmentation and the amino acid sequence, mainly the amino acids at or near the $\mathrm{N}$-terminus. After finding certain differences through statistical analysis of a large database of peptide MS/MS spectra, we synthesized specific peptides to study their fragmentation as a function of collision 
energy in an attempt to elucidate the mechanistic details. We find a significant difference in behavior when the N-terminus is either glutamine or glutamic acid compared with all other amino acids. The reason for this difference is rapid loss of water from $Q$ or $E$, leading to decreased basicity of the $\mathrm{N}$-terminus and easier transfer of its added proton away from the N-terminus.

\section{Experimental $^{1}$}

The peptides were synthesized with an AAPPTEC (Louisville, KY, USA) APEX 396 synthesizer by using standard procedures. The peptides were dissolved in methanol/water (vol:vol $=1: 1$ ) containing $0.1 \%$ formic acid. Electrospray ionization mass spectrometry was carried out with a Micromass (Waters Corp., Milford, MA, USA) Quattro Micro triple quadrupole instrument. First the mass spectrum was observed at different cone voltages to determine the voltage at which the protonated peptide ion peak is maximized. Then, selecting this precursor $(\mathrm{p})$ ion at that cone voltage into a collision cell (with 0.21 Pa (1.6 mTorr) Ar as collision gas), the MS/MS spectrum was measured at 20 different collision voltages. The range of collision voltage spanned from near zero up to a value where little precursor ion remained. The peak intensities of all the significant fragment ion peaks were calculated as a fraction from the total ion intensity and plotted as a function of collision voltage. Spectra were acquired in 'centroid' mode. Typically, relative $m / z$ values were within 0.2 of the theoretical $m / z$ values throughout the $m / z$ range of interest. For comparison, the spectra of several peptides were also measured with a linear ion trap instrument (LTQ; Thermo Electron Corp., Waltham, MA, USA) at a normalized collision energy setting of $35 \%$ of the maximum.

\section{Results and Discussion}

Recent reports of principal component analysis of a mass spectral database of diprotonated peptide ions revealed two types of fragmentation behavior [2], one leading to predominant formation of $b_{2}$ and $y_{n-2}$ fragments and the other showing minimal formation of these ions and greater fragmentation closer to the center of the peptide. A more recent study on several synthetic (Ala) $)_{x}$ His peptides showed decreased relative intensities of $b_{2} / y_{n-2}$ ions with increasing peptide length [4]. To further examine the distinction between these two groups of peptides, we analyzed protonated peptide ions in six libraries of peptide mass spectra: human,

\footnotetext{
${ }^{1}$ Certain commercial equipment, instruments, or materials are identified in this document. Such identification does not imply recommendation or endorsement by the National Institute of Standards and Technology, nor does it imply that the products identified are necessarily the best available for the purpose.
}

yeast, E. coli, mouse, Drosophila, and C. elegans. These libraries contained 432,446 MS/MS spectra of tryptic peptide ions composed of $\leq 20$ residues in charge state $1+(12 \%), 2+(65 \%)$, or $3+(22 \%)$, all with $\mathrm{K}$ or $\mathrm{R}$ at the C-terminus. Many of these spectra contained certain $y$ ions in two charged states, $\mathrm{y}_{n-\mathrm{x}}^{1+}$ and $\mathrm{y}_{n-\mathrm{x}}^{2+}$. The relative intensity of the singly and doubly charged ions can strongly depend on the specific residues adjacent to the breaking bond, and in some cases the doubly charged form predominates. The sum of both peak intensities is used in the following plots and is denoted simply as $\mathrm{y}_{n-\mathrm{x}}$. Plots of different $\mathrm{y}$ ion intensities as a function of peptide length (Figure 1) show the dominance of $y_{n-2}$ ions over other y ions. For diprotonated peptides containing one basic residue ( $\mathrm{R}$ or $\mathrm{K}$, at the C-terminus), $\mathrm{y}_{n-2}$ is very dominant for peptides with $<12$ residues (Figure 1a). The effect is somewhat similar in diprotonated peptides containing two basic residues (Figure $1 b$, where $\mathrm{R}, \mathrm{K}$, or $\mathrm{H}$ are the basic residues). Triprotonated peptides with one basic residue show dominant $\mathrm{y}_{n-2}$ for the longer peptides (Figure 1c), but peptides with only seven residues exhibit more intense $\mathrm{y}_{n-1}$ ion peaks. Monoprotonated peptides with one basic residue (no "mobile" protons [7, 8] show $\mathrm{y}_{n-2}$ only slightly higher than the other y ions (Figure 1d).

An example of the distribution of the above statistical results is plotted in Figure 2 for the 10,658 peptide ions containing 10 residues (tryptic, diprotonated, one basic amino acid). For each y ion, the different fractional intensities were divided into 20 bins, each differing by a factor of 2, and the number of peptide ions in each bin is plotted against bin number (expressed as percent from the maximum value). A logarithmic plot is chosen to show greater separation among the low intensity bins. It is clear from the plot that $y_{8}$ is the dominant peak in at least $70 \%$ of the spectra.

Deeper examination of the above general results shows that the tendency to produce dominant $\mathrm{y}_{n-2}$ ions also depends on the N-terminal residue (Figure 3 ). No systematic reduction of $\mathrm{y}_{n-2}$ or unexpected trends were found for specific residues at the second or third position from the N-terminus (data not shown). Whereas most N-terminal residues lead to similar behavior, E and $Q$ lead to much smaller intensities for the $\mathrm{y}_{n-2}$ ion peaks, but the difference decreases with increased peptide length (as reported before for a specific example [4]). Since N-terminal E and Q can undergo loss of water or ammonia to form pyroglutamic acid $[9,10]$, we examined the importance of these losses. It has been reported that tryptic peptides with N-terminal glutamine undergo loss of either water or ammonia when singly protonated, but predominantly loss of water when doubly protonated [10]. A plot of peak intensities due to both losses in monoprotonated peptides shows the prevalence of these losses with N-terminal E, Q, and $\mathrm{H}$ over all other residues (Figure $4 \mathrm{~b}$ ). However, a plot for diprotonated peptides (with one "mobile" proton) shows that water loss from N-terminal $Q$ is highly pronounced but the behavior of $\mathrm{E}$ falls within the range 


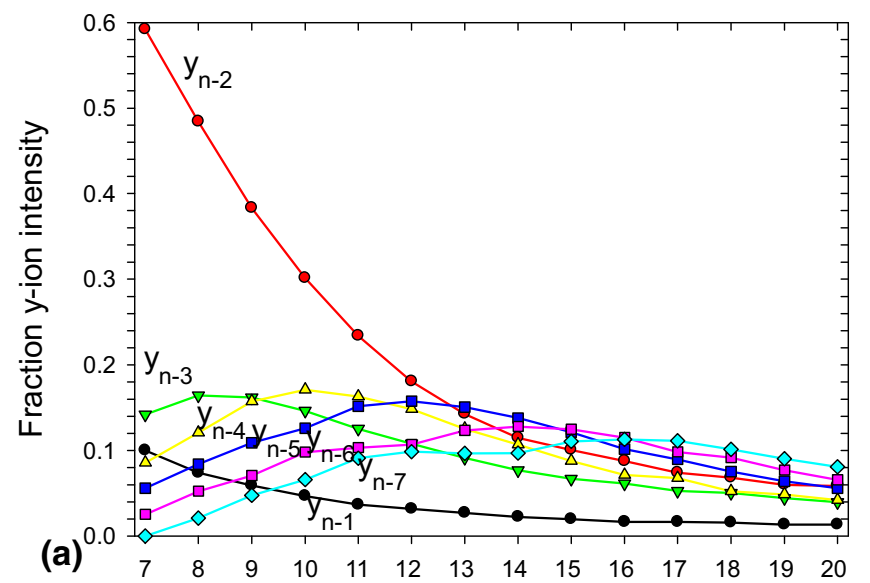

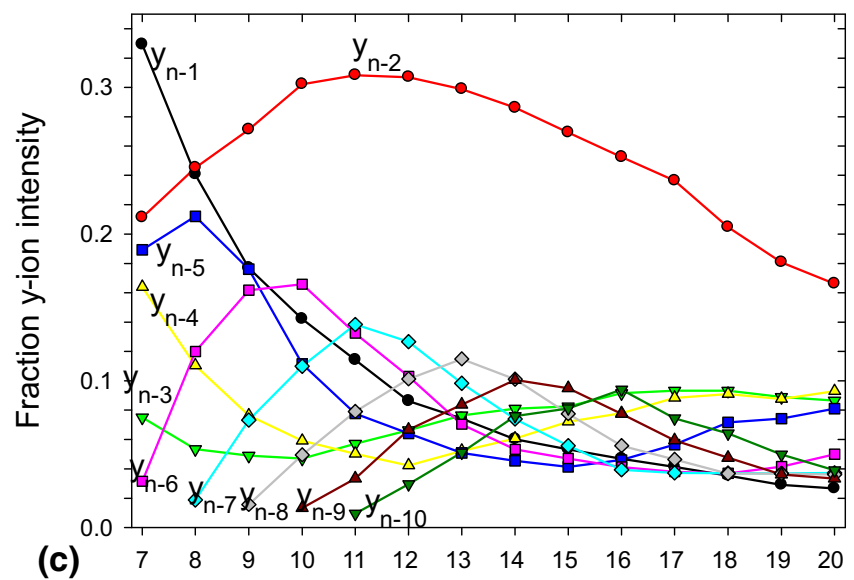

Peptide length (number of amino acid residues)

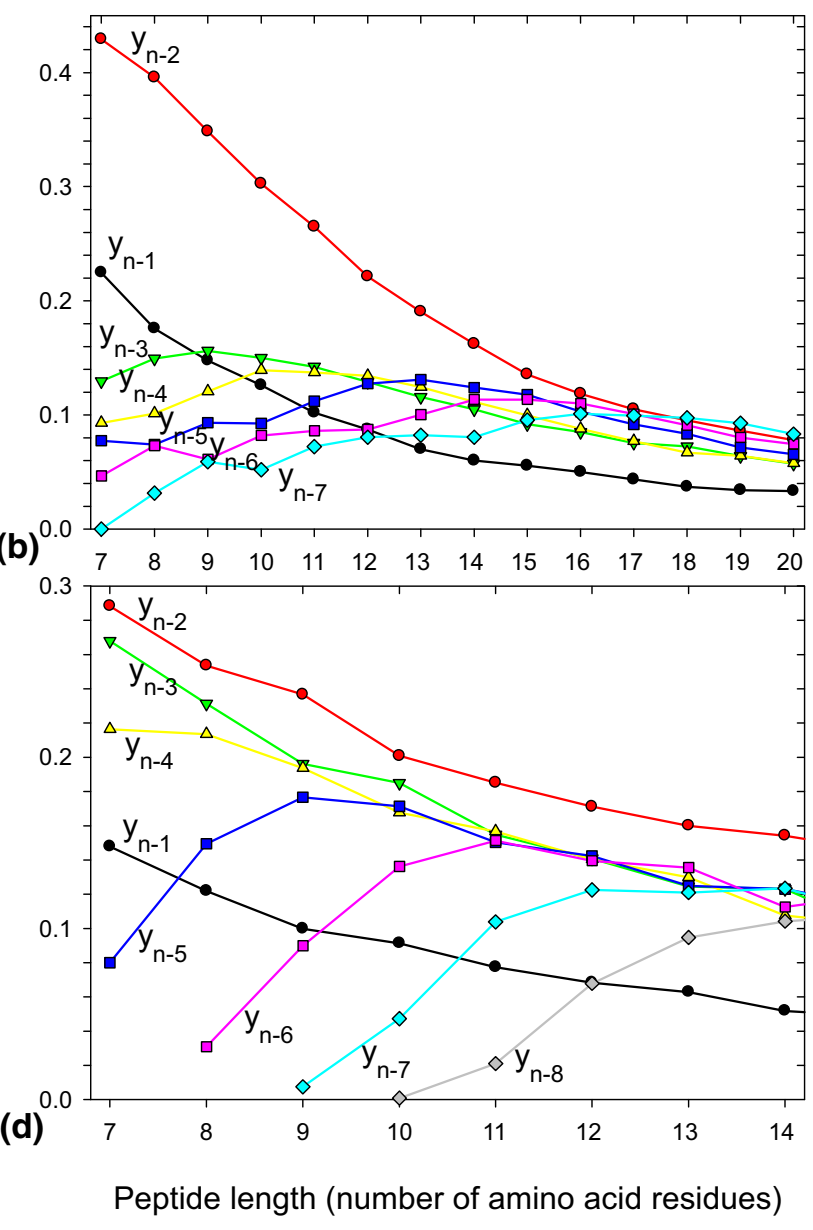

Figure 1. Fractions of y ion intensities as a function of peptide length (number of amino acids) for (a) diprotonated ions containing one basic amino acid, (b) diprotonated ions containing two basic amino acids, (c) triprotonated ions containing 2 basic amino acids, and (d) monoprotonated ions containing one basic amino acid. Intensities are the sums of charge states for each y ion.

of all other residues (Figure 4a). Likely the predominant loss of water from N-terminal $\mathrm{Q}$ in diprotonated peptide ions may be the cause for the greatly diminished formation of $\mathrm{y}_{n-2}$ ions in such peptides. This supposition, however, does not explain the behavior of peptides with N-terminal E.

To elucidate the mechanism for the different behavior of N-terminal glutamine and glutamic acid compared with all other amino acids, peptides were synthesized with variations in peptide length (5 to 10 residues), $\mathrm{C}$-terminal $\mathrm{K}$ or $\mathrm{R}$, and various $\mathrm{N}$-terminal residues, including pyroglutamic acid and $\mathrm{N}$-acetyl derivatives. Representative MS/MS spectra of diprotonated peptide ions are compared in Figure 5. LALVFAYK shows $\mathrm{y}_{6}$ as the most intense peak and $b_{2}$ as the most intense of the $b$ ions. The relative intensity of the $\mathrm{y}_{6}$ ion peak is greater in the linear ion trap mass spectrometer (bottom spectrum) than in the triple quadrupole instrument (top spectrum) because of differences in collision energies, but the general trends are the same. Similar trends were found when N-terminal L was replaced with $\mathrm{A}, \mathrm{N}$, or $\mathrm{P}$ (not shown). With N-terminal E, however, the most intense peak is $\mathrm{y}_{4}$, while $\mathrm{y}_{6}$ is much weaker in both mass spectrometers.
With N-terminal $\mathrm{Q}$ also $\mathrm{y}_{6}$ is much weaker than $\mathrm{y}_{4}$ but the dominant peak is that due to loss of water from the precursor ion $(\mathrm{p}-\mathrm{w})$.

Detailed comparison of the fragmentation of these ions is shown by the collision voltage dependence of their fragment peak intensities (Figure 6). For diprotonated ions, MS/MS spectra were recorded at 20 collision voltages between 1 and $20 \mathrm{~V}$, showing the gradual formation of each fragment with increasing voltage and its decrease at higher voltage due to further fragmentation. In Figure 6, only the range from 7 to $17 \mathrm{~V}$ is presented and only the most intense peaks are shown. With diprotonated LALVFAYK, the $\mathrm{y}_{6}$ ion dominates at low collision voltage and remains dominant throughout the presented range. Similar behavior was observed when N-terminal L was replaced with $\mathrm{A}, \mathrm{N}$, or $\mathrm{P}$ (not shown). With N-terminal $\mathrm{E}$, however, $\mathrm{y}_{4}$ clearly dominates over $\mathrm{y}_{6}$ and $y_{5}$, and in addition, a peak due to water loss $(p-w)$ is clearly evident at low collision voltage. This indicates that the diprotonated EALVFAYK ion undergoes water loss before significant peptide bond fragmentation and this water loss leads to different selectivity in peptide bond cleavage. The $\mathrm{p}-\mathrm{w}$ peak is much more pronounced in QALVFAYK than in EALVFAYK and persists at higher 


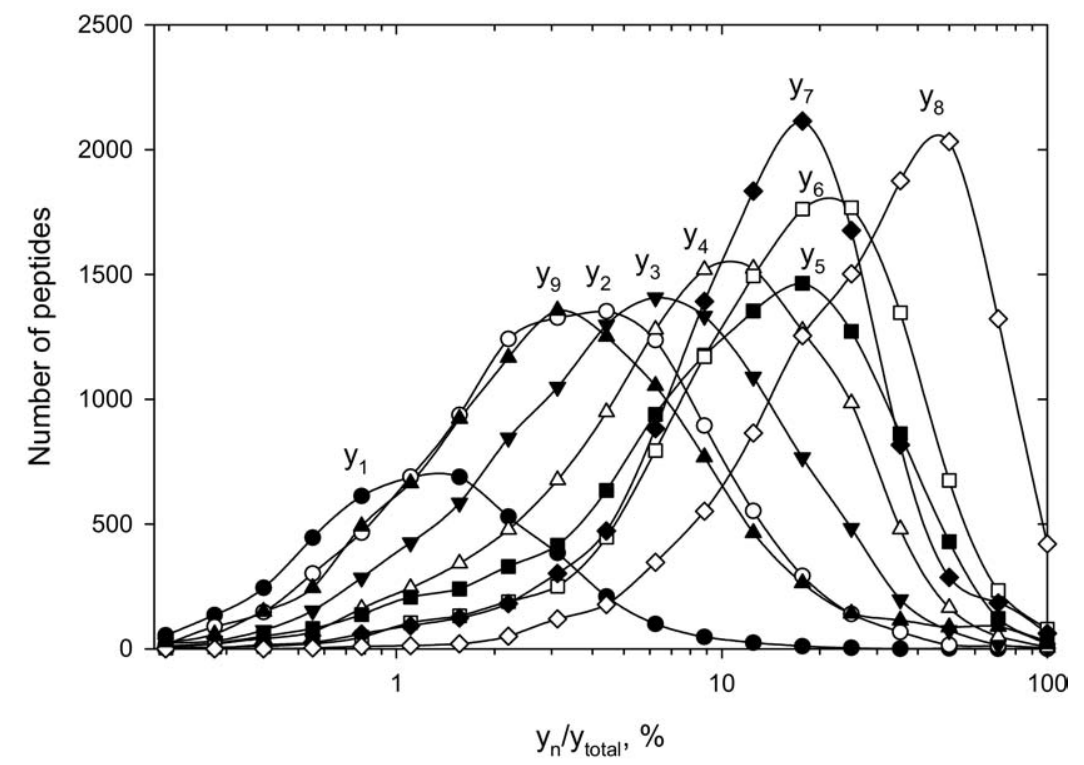

Figure 2. Distribution of peptide ions showing different fractional intensities for the different y ions. (Plot only for diprotonated tryptic peptides containing 10 residues and no additional basic residue, intensities are sums of charge states).

collision voltage, i.e., requires more energy to undergo fragmentation. Similar trends for the y ions were observed with peptides containing five to 10 amino acid residues, with C-terminal K or R. All peptides with N-terminal E or $\mathrm{Q}$ undergo water loss at low collision voltage, before any peptide bond fragmentation is apparent. This water loss is followed by peptide bond fragmentation at various sites, with no preference to the $b_{2} / y_{n-2}$ site, which is the "normal" site for most other peptides.

The main peaks in the MS/MS spectra of the diprotonated ions of 192 peptides are listed in their approximate order of intensity in the Supplementary Table S1, which can be found in the electronic version of this article. Most peptides exhibit the "normal" fragmentation to $b_{2}$ and $y_{n-2}$ ions, with varying relative intensities. In some cases, additional peaks are also intense but rarely are they more intense than the $y_{n-2}$ or $b_{2}$ ions. In a few cases, $y_{n-2}$ is not the most intense peak, either because it is split into two peaks, singly protonated (denoted $\mathrm{y}_{n-2}$ ) and doubly protonated (denoted $\mathrm{y}_{n-2}^{2+}$ ), or because particular amino acid residues promote other fragmentation routes (see below). Peptides with $\mathrm{N}$ terminal $Q$ exhibit a dominant water-loss peak $(p-w)$ at all collision voltages. Peptides with N-terminal E ex-

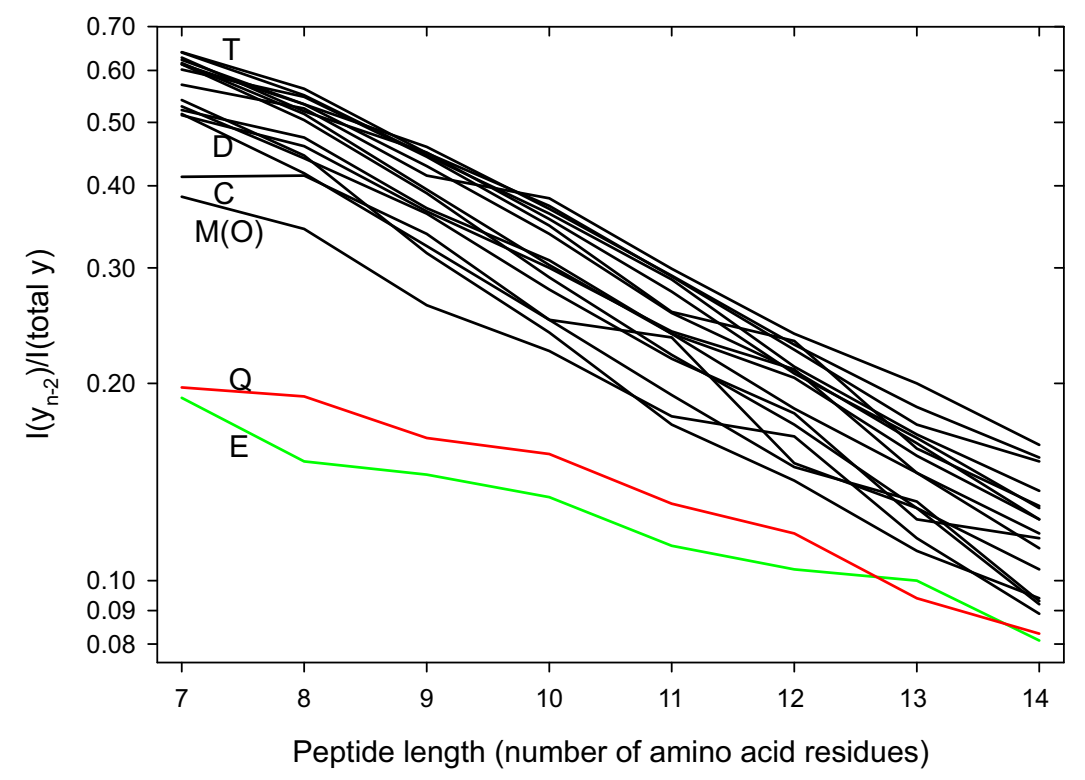

Figure 3. Fractional intensities of $\mathrm{y}_{n-2}$ ions as a function of peptide length for peptides with different $\mathrm{N}$-terminal residues (intensities are sums of charge states, $\mathrm{y}_{n-2}$ and $\mathrm{y}_{n-2}^{2+}$ ). 


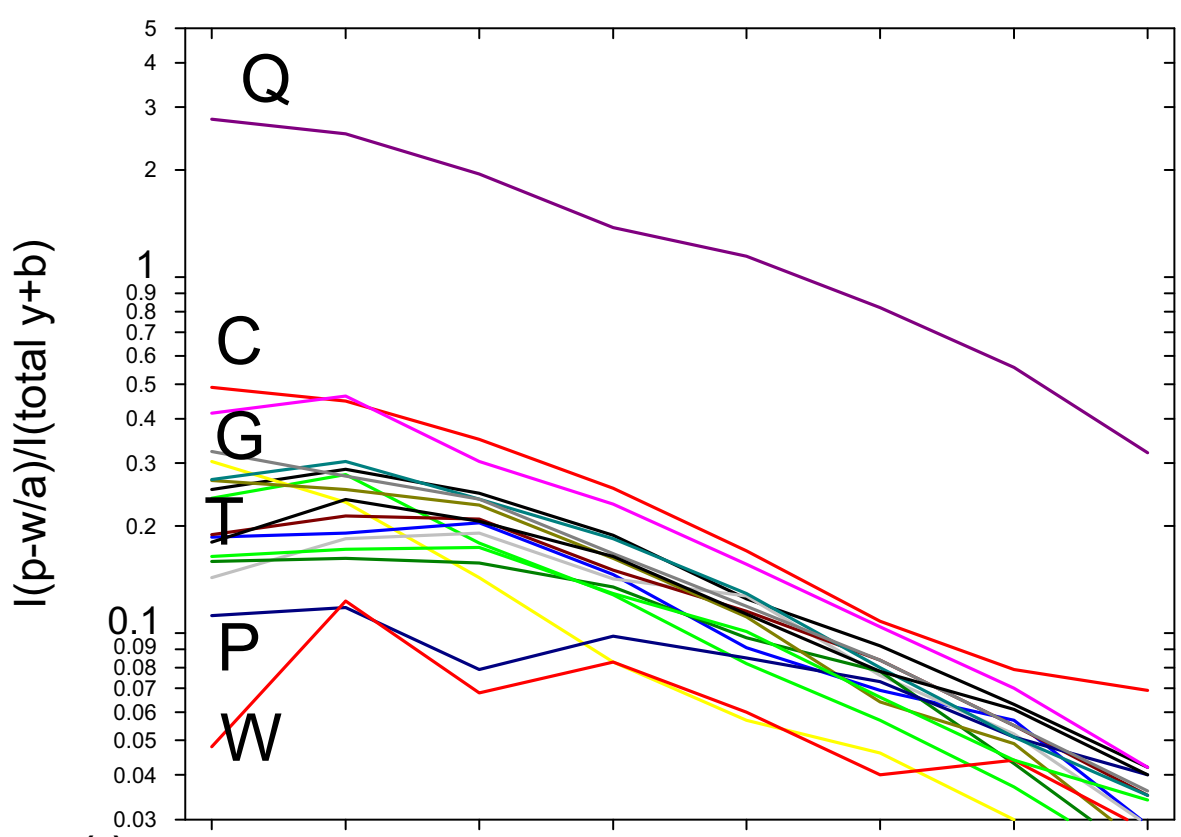

(a)

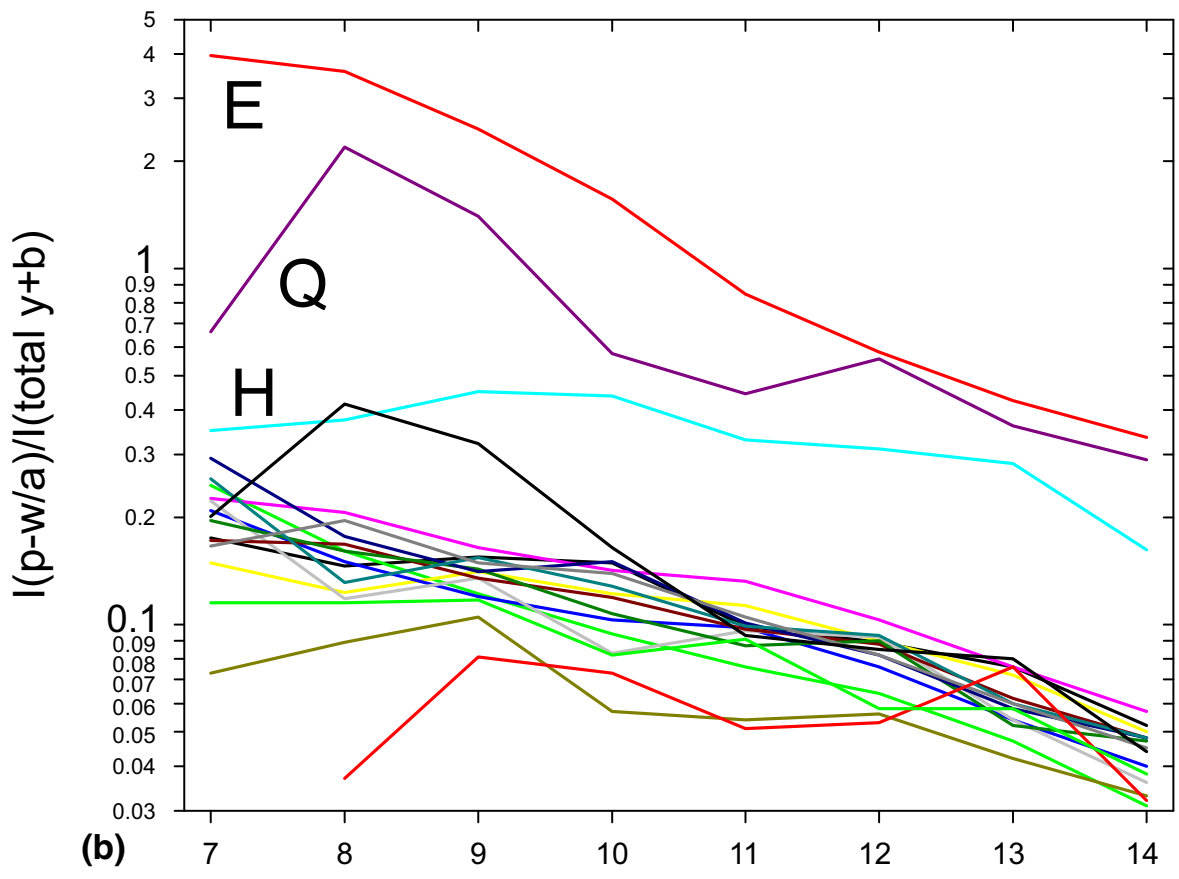

Peptide length (number of amino acid residues)

Figure 4. Fractional intensities of water/ammonia loss ions as a function of peptide length for peptides with different $\mathrm{N}$-terminal residues. (a) Diprotonated peptides, and (b) monoprotonated peptides, all with a single basic residue. (Intensities are sums of charge states).

hibit a water-loss peak at low collision voltage, but this peak is small at high collision voltage. These two groups of peptides do not exhibit the "normal" $b_{2} / y_{n-2}$ fragmentation pattern.

To investigate whether the deviant behavior of peptides with $\mathrm{N}$-terminal $\mathrm{E}$ or $\mathrm{Q}$ is a result of water-loss from these residues, we synthesized three peptides with $\mathrm{N}$-terminal pyroglutamic acid. These peptides do not undergo loss of water, but their dominant peaks are the same as those observed with the corresponding peptides with $\mathrm{N}$-terminal glutamic acid or glutamine. These findings indicate that peptides with $\mathrm{N}$-terminal $\mathrm{E}$ or $\mathrm{Q}$ do not fragment via the "normal" pathway because they preferentially undergo water loss, leading to alteration in the behavior of the resulting ions. Loss of water from $\mathrm{E}$ results in cyclization of the terminal $\mathrm{NH}_{2}$ with 


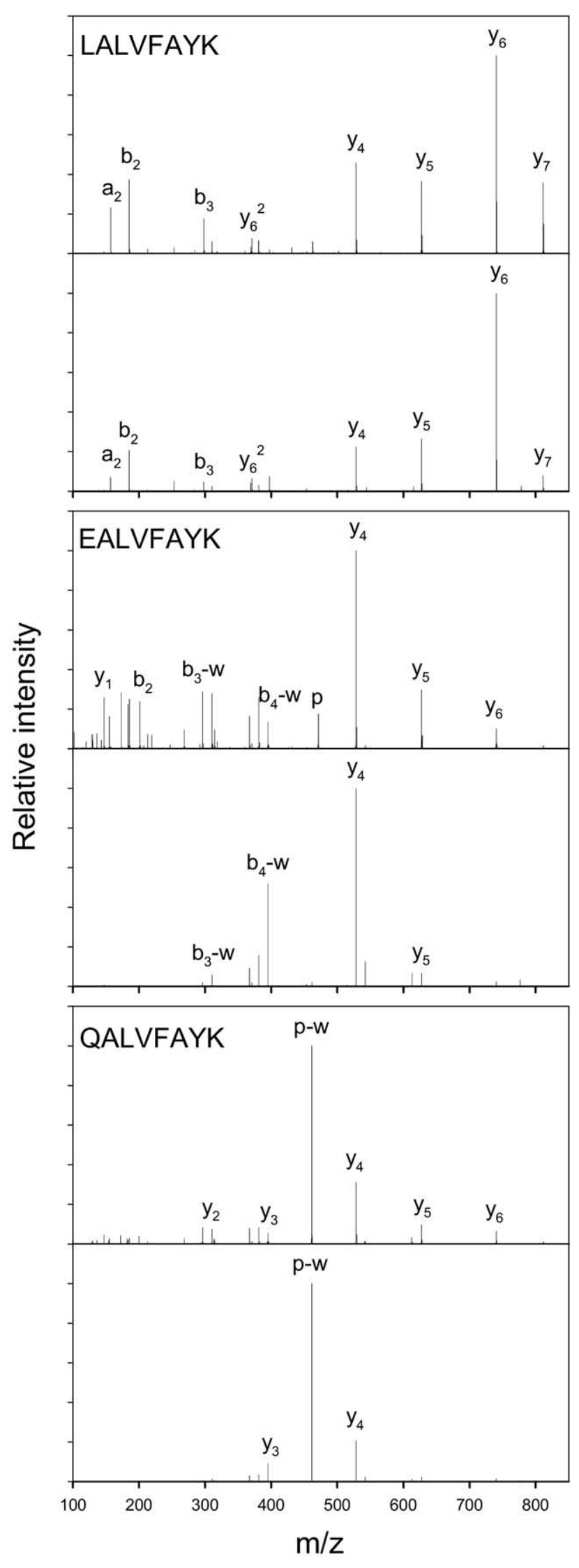

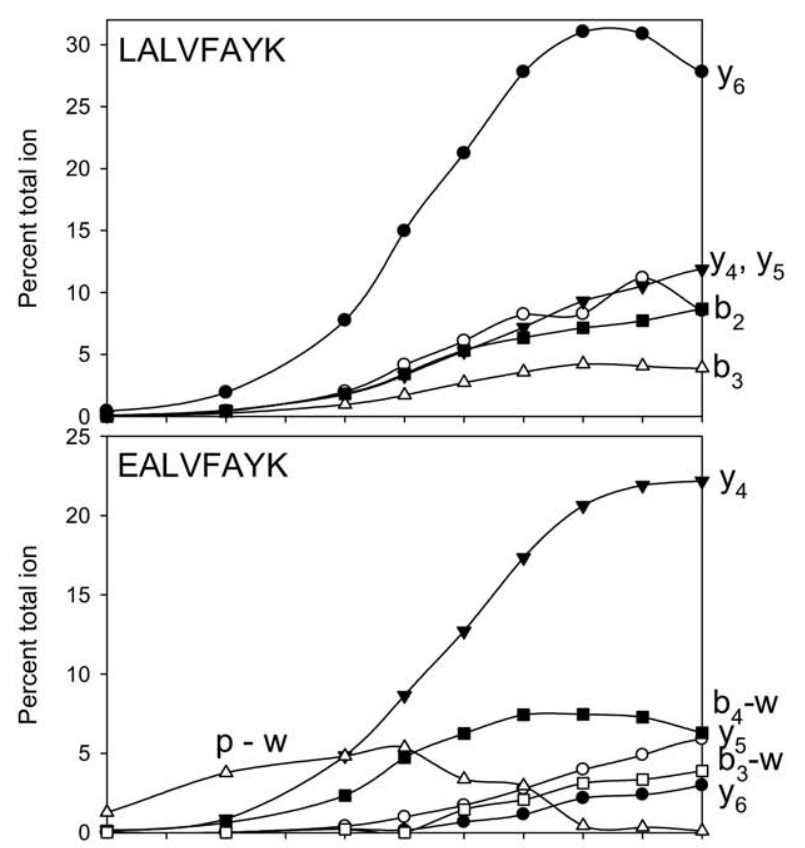

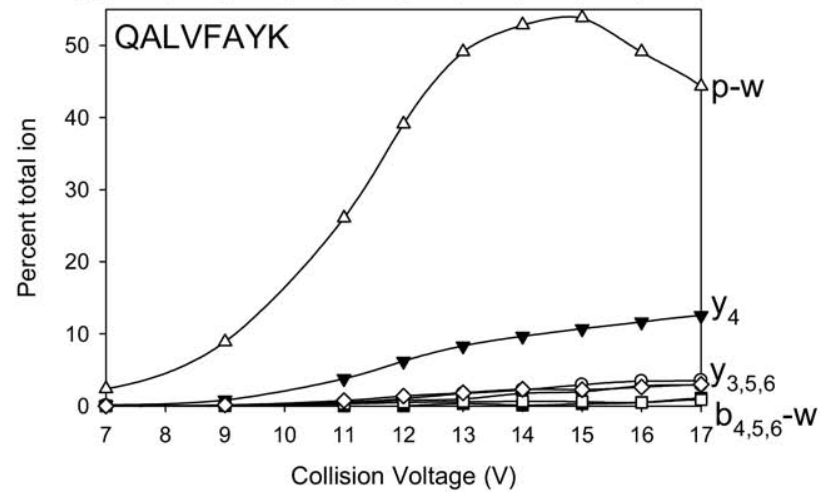

Figure 6. Peak intensity as a function of collision voltage in the MS/MS spectra of diprotonated ions of LALVFAYK, EALVFAYK, and QALVFAYK recorded with the Micromass instrument.

the side-chain carboxyl group to form the pyroglutamic acid residue (Structure 3) [11].<smiles>CC(=O)C1CCC(=O)N1</smiles>

3<smiles>[NH][C@H]1CC[C@@H](C(=O)O)N1</smiles>

4
In this ring, the strongly basic $\mathrm{NH}_{2}$ group has been converted into a much less basic amide function. As a result, the second proton on the peptide is no longer localized at the $\mathrm{N}$-terminus and can easily move to the

Figure 5. MS/MS spectra of diprotonated ions of LALVFAYK, EALVFAYK, and QALVFAYK. Top spectra were recorded with the Micromass triple quadrupole mass spectrometer at collision voltages of 17,23 , and $18 \mathrm{~V}$, respectively. Bottom spectra were recorded with the LTQ ion trap instrument using a collision energy setting of $35 \%$ from the maximum. 
various peptide bonds, leading to fragmentation at various positions. With $\mathrm{N}$-terminal $\mathrm{Q}$ the situation is somewhat similar. Q-terminated peptides are known to undergo loss of either water or ammonia when singly protonated but predominantly loss of water when doubly protonated [10]. In the latter case, the product is similar to pyroglutamic acid except with $\mathrm{NH}$ instead of $\mathrm{O}$ on the ring (Structure 4). In this structure also the terminal $\mathrm{NH}_{2}$ group is converted to a much less basic amide, leading to the different behavior. If the cause of this "abnormal" behavior of terminal $\mathrm{E}$ and $\mathrm{Q}$ is the result of conversion of the terminal basic $\mathrm{NH}_{2}$ group into a much weaker base, we expect to find the same effect when the terminal $\mathrm{NH}_{2}$ is acetylated. Indeed, three acetylated peptides (Table S1) demonstrate this behavior. These results indicate that most diprotonated peptide ions dissociate into $\mathrm{b}_{2}$ and $\mathrm{y}_{n-2}$ ions via $\mathrm{a}$ mechanism similar to that outlined in Scheme 3 of reference [3]. The two protons are localized predominantly at the $\mathrm{C}$-terminal $\mathrm{K}$ or $\mathrm{R}$ and the $\mathrm{N}$-terminal amino group, because of the higher basicities of these sites and because of charge repulsion. The presence of the proton at the $\mathrm{N}$-terminus promotes formation of $b_{2}$ ions, leaving $\mathrm{y}_{n-2}$ as the remaining ion. However, when the amino group at the N-terminus is converted into an amide function, by acetylation or cyclization, and its proton affinity greatly diminishes, the second proton on the peptide ion is no longer localized at this site. It becomes more "mobile" and thus can effect dissociation at various peptide bonds with no preference to formation of $b_{2}$.

From the above discussion it may be concluded that diprotonated tryptic peptide ions with five to 10 residues and only one basic amino acid exhibit the "normal" behavior of producing the $b_{2} / y_{n-2}$ ions as the dominant peaks in their spectra, except for those peptides with N-terminal Q or E, which exhibit the "abnormal" behavior. This conclusion, however, is true for most, but not all, peptides. From the results in Table S1 (and additional results obtained in this laboratory) it appears that specific amino acid residues in specific positions can have profound effects on the spectra. First of all, proline strongly enhances fragmentation at its N-terminus. When $\mathrm{P}$ is in the third position (from the N-terminus), formation of $\mathrm{y}_{n-2}$ is greatly enhanced. Very often, both $\mathrm{y}_{n-2}$ and $\mathrm{y}_{n-2}^{2+}$ are observed because $\mathrm{P}$ is slightly more basic than many other residues. This enhancement by P overcomes the effect of $E$ and $Q$ and changes the behavior of peptides N-terminated with $\mathrm{E}$ or Q from "abnormal" to "normal". Peptides with P in the second position tend to form dominant peaks of $\mathrm{y}_{n-1}^{2+}$ ions, thus counteracting the "normal" behavior of many peptides. $\mathrm{P}$ at the $\mathrm{N}$-terminus behaves like many other amino acid residues. Histidine, which is a stronger base than proline, also affects the fragmentation behavior in a similar manner, depending on its position within the peptide sequence.

Other effects are apparent for the aliphatic hydrophobic amino acids, most pronounced with V. In many peptides the $\mathrm{V}$ residue promotes fragmentation at its C-terminus; I and L appear to have similar but weaker effects. For example, the peptide EVFGYLAFK fragments predominantly to $\mathrm{y}_{n-2}$ and $\mathrm{y}_{n-2}^{2+}$, i.e., the effect of $\mathrm{V}$ counteracts the effect of $\mathrm{E}$ and leads to "normal" fragmentation in this case, despite the presence of $E$ terminus. In many other cases, fragmentations at the C-termini of V, I, or L lead to enhanced peak intensities, sometimes counteracting the "normal" pattern. Finally, by contrast with the water loss from E and Q, it is noted that water loss from N-terminal ST or TT combinations [12], despite giving a dominant $\mathrm{p}-\mathrm{w}$ peak, does not alter the dominance of $b_{2} / y_{n-2}$ among the rest of the peaks. Water losses from terminal S, T, or D also have no effect of the fragmentation pattern. These water losses do not involve the terminal $\mathrm{NH}_{2}$ group and do not modify its basicity.

To derive a quantitative measure of the "normal" and "abnormal" behavior discussed above, we calculated the sum of the intensities of the $\mathrm{b}_{2}, \mathrm{y}_{n-2}$, and $\mathrm{y}_{n-2}^{2+}$ ions as percent of the total intensities of all ions at a collision voltage $V_{1 / 2}$. The value of $V_{1 / 2}$ is the voltage at which the sum of intensities of all fragment peaks is equal to the remaining intensity of the precursor ion, i.e., when approximately half the precursor ions underwent dissociation. The reason for choosing this value rather than a constant collision voltage is that the energy required to fragment the peptide ion depends on its mass, charge, and specific amino acid basicities [13]. The percentages calculated for different peptides vary greatly but their averages show clear differences between the different groups of peptides. For 22 peptides with $\mathrm{N}$-terminal $\mathrm{E}$, and not containing $\mathrm{P}$, the average value is $\sim 4.1 \%$, for three peptides with terminal $\mathrm{Q}$ the value is $4.4 \%$, and for three acetylated peptides the value is $5.1 \%$. On the other hand, for 108 other peptides not terminated with $\mathrm{E}, \mathrm{Q}$, or acetyl group the average value is $21 \%$, i.e., four to five times higher, with wide variations due to peptide length and specific amino acid effects.

In summary, most doubly protonated tryptic peptide ions dissociate to form two singly protonated ions, $b_{2}$ and $\mathrm{y}_{n-2}$. The two protons on these peptide ions are localized initially at the two termini, which are also the most basic sites, i.e., the C-terminal $\mathrm{K}$ or $\mathrm{R}$ and $\mathrm{N}$ terminal $\mathrm{NH}_{2}$ group. This separation is further promoted by charge repulsion. The presence of the proton at the N-terminus and the relative stability of the $b_{2}$ ion promote the fragmentation to $b_{2} / y_{n-2}$. In many cases, doubly protonated $\mathrm{y}_{n-2}^{2+}$ ions are formed, leaving a neutral $b_{2}$ fragment. When the terminal amine group is converted to a much less basic amide group, either by acetylation or by cyclization with the side-chain of E or $Q$, the second proton on the peptide ion is no longer localized at the N-terminus. The proton affinity of the terminal amide is expected to be similar to that of internal peptide bonds and thus the proton may be initially localized at any peptide bond. Only charge repulsion prevents it from localizing close to the C- 
terminus. In such cases, formation of $\mathrm{y}_{n-2}$ is no longer dominant and smaller y ions are observed instead. The $b_{2}$ ions formed from both groups of peptides have been shown $[5,6]$ to primarily generate the protonated oxazolone structure. Their formation is not inhibited by the cyclization of N-terminal E or Q just as it is not inhibited by the presence of the cyclic proline at the N-terminus. Instead, it is inhibited by the loss of basicity due to cyclization or acetylation. Cyclization of $\mathrm{E}$ and $Q$ is a result of loss of water between the terminal amino group and the side-chain carboxyl or carbamido group. Other neutral losses, such as loss of $\mathrm{H}_{2} \mathrm{O}$ from $\mathrm{T}$ or $\mathrm{S}$ or loss of $\mathrm{CH}_{3} \mathrm{SOH}$ from oxidized $\mathrm{M}$, which do not affect the basicity of the terminal amine, exert no effect on the fragmentation route. When the peptides are longer, mainly above 10 residues, the dominance of $\mathrm{b}_{2} / \mathrm{y}_{n-2}\left(\right.$ or $\left.\mathrm{b}_{2} / \mathrm{y}_{n-2}+\mathrm{y}_{n-2}^{2+}\right)$ diminishes because there are more peptide bonds to compete for the proton with the terminal amino group and because charge repulsion becomes smaller. And finally, these general trends are sometimes reversed by the effects of specific amino acids in specific positions as discussed above.

\section{Appendix A Supplementary Material}

Supplementary material associated with this article may be found in the online version at doi:10.1016/ j.jasms.2010.03.027.

\section{References}

1. Aebersold, R.; Goodlett, D. R. Mass Spectrometry in Proteomics. Chem. Rev. 2001, 101, 269-295.

2. Savitski, M. M.; Fälth, M.; Fung, Y. M. E.; Adams, C. M.; Zubarev, R. A. Bifurcating Fragmentation Behavior of Gas-Phase Tryptic Peptide Dications in Collisional Activation. J. Am. Soc. Mass Spectrom. 2008, 19, 1755-1763.

3. Paizs, B.; Suhai, S. Fragmentation Pathways of Protonated Peptides. Mass Spectrom. Rev. 2005, 24, 508-548.

4. Harrison, A. Charge-Separation Reactions of Doubly-Protonated Peptides: Effect of Peptide Chain Length. J. Am. Soc. Mass Spectrom. 2009, 20, 1890-1895.

5. Bythell, B. J.; Somogyi, A.; Paizs, B. What is the Structure of $b_{2}$ Ions Generated from Doubly Protonated Tryptic Peptides? J. Am. Soc. Mass Spectrom. 2009, 20, 618-624.

6. Bythell, B. J.; Erlekam, U.; Paizs, B.; Maître, P. Infrared Spectroscopy of Fragments from Doubly Protonated Tryptic Peptides. Chem. Phys. Chem. 2009, 10, 883-885.

7. Wysocki, V. H.; Tsaprailis, G.; Smith, L. L.; Breci, L. A. Mobile and Localized Protons: A Framework for Understanding Peptide Dissociation. J. Mass Spectrom. 2000, 35, 1399-1406.

8. Dongré, A. R.; Jones, J. L.; Somogyi, Á.; Wysocki, V. H. Influence of Peptide Composition, Gas-Phase Basicity, and Chemical Modification on Fragmentation Efficiency: Evidence for the Mobile Proton Model. J. Am. Chem. Soc. 1996, 118, 8365-8374.

9. Harrison, A. Fragmentation Reactions of Protonated Peptides Containing Glutamine or Glutamic Acid. J. Mass Spectrom. 2003, 38, 174-187.

10. Neta, P.; Pu, Q.-L.; Kilpatrick, L.; Yang, X.; Stein, S. E. Dehydration Versus Deamination of N-Terminal Glutamine in Collision-Induced Dissociation of Protonated Peptides. J. Am. Soc. Mass Spectrom. 2007, 18, 27-36.

11. Harrison, A. G. Ion Chemistry of Protonated Glutamic Acid Derivatives. Int. J. Mass Spectrom. 2001, 210/211, 361-370.

12. Neta, P.; Pu, Q. L.; Yang, X.; Stein, S. E. Consecutive Neutral Losses of $\mathrm{H}_{2} \mathrm{O}$ and $\mathrm{C}_{2} \mathrm{H}_{4} \mathrm{O}$ from N-Terminal Thr-Thr and Thr-Ser in CollisionInduced Dissociation of Protonated Peptides. Position-Dependent Water Loss from Single Thr or Ser. Int. J. Mass Spectrom. 2007, 267, 295-301.

13. Neta, P.; Simón-Manso, Y.; Yang, X.; Stein, S. E. Collisional Energy Dependence of Peptide Ion Fragmentation. J. Am. Soc. Mass Spectrom. 2009, 20, 469-476. 